\title{
Determining the Wood (Parashorea spp.) Decaying and Metal Corroding Abilities of Eight Fungi ${ }^{1}$
}

\author{
Sihati SUPRAPTI ${ }^{2} \cdot$ DJARWANTO $^{2} \cdot$ Listya Mustika DEWI (iD ${ }^{2, \dagger}$
}

\begin{abstract}
Fungi are wood-decaying organisms, and this is an important trait that should be considered in wood utilization. When fungi attack wood, it decreases the quality of the wood. The use of metal screws has become an important part of woodworking. The ability of fungi to decay wood and damage metal screws that are embedded into wood is varied. In this study, eight fungal species were evaluated with respect to their ability to decay Parashorea smythiesii and $P$. tomentella wood. In addition, the effect of fungi on corroding metal screws was determined using the Kolle flask method. The evaluation showed that the fungal species Schizophyllum commune, Pycnoporus sanguineus, and Polyporus arcularius were highly capable of decaying Parashorea spp. woods. The greatest wood weight loss occurred with the heartwood of $P$. tomentella exposed to $S$. commune. Based on the classification of wood resistance against fungal attack, the two Parashorea spp. were classified as moderately resistant woods (class III). Schizophyllum commune was classified as highly capable of decaying wood that was embedded with metal screws and was highly capable of corroding metal screws placed in fungi-culture media. The greatest weight of rust powder formed because of screw corrosion was obtained from screw-embedded wood exposed to S. commune. Additionally, the most severe corrosion of metal screws that were embedded into woods was caused by the activities of $P$. arcularius. Moreover, the average weight loss of screw-embedded wood was greater than that of unscrewed wood.
\end{abstract}

Keywords: wood-decaying fungi, wood resistance, metal screw corrosion, Parashorea smythiesii, Parashorea tomentella

\section{INTRODUCTION}

Indonesia has an abundant number of fungi in nature that has functional benefit for the environment. 'Fungi are recognized to be notable organisms that create a broad range of biological products in the form of secondary metabolites (Lee et al., 2016). For example, Polyporus brumalis, the white-rot fungus that able to produce terpenoids through terpene synthase, which catalyzes an essential step that forms an enormous range of sesquiterpene products from farnesyl pyrophosphate (An et al., 2018). On the other hand, fungi have become one of the most crucial wood decay organisms that should be considered in the utilization of wood. Many types of fungi have been determined in affecting the durability of wood in a certain condition. The wellknown types of fungi that attack wood are soft rot, brown rot, and white rot. The type of decay could

\footnotetext{
${ }^{1}$ Date Received November 11, 2019, Date Accepted December 25, 2019

${ }^{2}$ Forest Products Research and Development Center, Jl. Gunung Batu No. 5, Bogor 16610, West Java, Indonesia

† Corresponding author: Listya Mustika DEWI (e-mail: lizthya@gmail.com, ORCID: 0000-0003-4517-0196)
} 
classify cell wall degradation caused by fungal attack. Every type of fungi produce various enzymes and degrade different woody materials and thus inhabit ecological functions (Pawlik et al., 2019).Therefore, assessing the durability of wood against fungi is important to determine the proper utilization of wood and also possible treatment to enhance the durability of wood.

Parashorea is one of the genera from the Dipterocarpaceae family that consists of about 14 species, which are widely distributed throughout the South-East Asia region. In Indonesia, 9 species of Parashorea distributed in Sumatra (3 species) and Kalimantan islands (6 species) (Soerianegara and Lemmens, 1993). While Oey (1990) reported the basic properties i.e. specific gravity, strength class and durability class of four Parashorea wood species in Indonesia. Parashorea has been considered as one of the main commercial timbers in South-East Asia. However, some species have not been studied comprehensively for their basic properties. This study was focused on observing two species of Parashorea from East Kalimantan i.e. Parashorea smythiesii and $P$. tomentella for their resistance against fungi attack.

In the utilizations of wood such as for building construction, wood often incorporates with metals such as nails and screws. In particular condition, the metal can be damaged through corrosion process because of direct physical contacts between woods and metals especially under wet environments ( $\mathrm{Li}$ et al, 2011; Sugiyanto and Sudika, 2010). The corrosion rate might be affected by the attack of fungi on wood, so that this study also investigates the effect of fungi in corroding metal screw that embedded into wood samples. This research is aimed to examine the capability of eight fungi species to decay Parashorea spp. wood originated from East Kalimantan at their heartwood and sapwood parts, and to examine the influence of selected fungi in corroding metals.

\section{MATERIALS and METHODS}

\subsection{Materials}

The main wood materials consisted of Parashorea smythiesii Wyatt-Smith ex P. Ashton and Parashorea tomentella (Sym.) Meijer. The wood samples were obtained from natural production forest grown in Berau, East Kalimantan, located at coordinate point $01^{\circ} 85^{\prime} 06.1^{\prime \prime}$ S, 117 $04^{\prime} 56.7^{\prime \prime}$ and $01^{\circ} 85^{\prime} 06.1^{\prime \prime}$ S, $117^{\circ} 04^{\prime} 55.3^{\prime \prime} \mathrm{E}$, respectively. The materials used were malt extract, bacto agar, potato dextrose agar (PDA), alcohol, distilled water, and metal screws. The tested fungi were eight species such as Chaetomium globossum FRI Japan-5-1, Lentinus lepideus HHBI-267, Polyporus arcularius HHBI-371, Polyporus sp. HHBI-209, Phlebia brevispora Mad., Pycnoporus sanguineus HHBI-324, Schizophyllum commune HHBI-204, and Tyromyces palustris HHBI-378.

\subsection{Methods}

The tested specimens of two Parashorea spp. were prepared in $5 \mathrm{~cm}$ (parallel to grain direction) $\mathrm{x} 2.5 \mathrm{~cm}$ (wide) x $1.5 \mathrm{~cm}$ (thick) size. The sample preparation followed the previous research by Djarwanto, Suprapti and Hutapea (2018); Suprapti and Djarwanto (2014). The specimens were taken from sapwood (near the cambium layer) and heartwood (nearest layer to the pith) at bottom, middle, and top of logs and tested for determining the properties of wood-resistance. Meanwhile, tested specimens for properties of corroding-wood action on screws were obtained from heartwood $(4 \mathrm{~cm}$ laterally from the pith), also at bottom, middle, and top of logs; and further embedded with screws. All test specimens were marked with numbers, weighed, and then oven-dried at $103 \pm 2^{\circ} \mathrm{C}$ until reached constant weight.

Test media for decaying-fungi were MEA (malt-extractagar), which consisted of malt-extract (3\%), bacto-agar 
(2\%), and distilled water. The media used for Chaetomium globosum fungi comprised PDA (potato-dextrose-agar) $39 \mathrm{~g} / \mathrm{l}$ in distilled water, similarly as performed by Suprapti, Djarwanto dan Andianto (2016). All the media after being inoculated with fungi-culture were stored in the incubation chamber until the growth of fungi mycelium became evenly spread and thickened, following Indonesia's National Standard (SNI, 2014).

The decay and corroding-wood properties test was performed using the Kolle-flask method, which referred to Indonesia's National Standard (SNI 7207:2014). The wood samples with their known oven-dry weight were put into the Kolle-flasks that had already contained the pure culture of fungi. Each of Kolle-flasks was allocated for three wood specimens, comprising sapwood and heartwood; and each was from consecutively bottom, middle, and top of the tree stem (decay test). For corroding test, each of those Kolle-flasks was exposed with three wood specimens, comprising unscrewedwood and screwed-wood; and each was from consecutively bottom, middle, and top of wood logs, and then incubated for 12 weeks. At the end of the tests, the samples were removed from the flasks, cleaned from the attached mycelium, weighed to acquire the wet weight of the decayed woods, and put into the oven for to reach oven-dry weight.

In another related experiment, as many as three Kolle-flasks that also already contained fungi-culture media were prepared for each of eight fungi species, whereby further on media inside the flasks were laid-down the two screws vertically upside down such that the screw heads touched the upper media surface; and then incubated for 12 weeks. After 12-weeks incubation, screws were removed from Kolle-flasks, then cleaned off the attaching mycelium. All of the screws in wood or fungi medium further washed with a technical alcohol-acetone solution with the aid of nylon brushes. Afterwards, the cleaned screws were allowed to dry, and further weighed to obtain the final screw weight, as has also done so in the previous research (Djarwanto and Suprapti, 2015).

The percentage of weight loss, calculated from initial and final weight, was used as a measure of the amount both of decay wood and corroding screw (SNI, 2014). As one of wood basic properties, initial $\mathrm{pH}$ of two wood species of Parashorea spp. were each measured on the mixture of powdered wood specimens and distilled water (1:1 proportion, w/w).

\subsection{Data analysis}

Data analysis on weight loss (WL, \%; this related to decay-resistance test) of Parashorea spp. woods were assessed using completely randomized design (CRD) with $2 \times 3 \times 2 \times 8$ factorial pattern (wood species, vertical wood part (top, middle, bottom), horizontal wood part [sapwood, heartwood] in the logs, and fungi species) with 6 replicates; and also using CRD (for WL; besides, this related to corroding-wood action test) with $2 \times 3 \times 2 \times 8$ factorial pattern (wood species, vertical wood part [upper/top, middle, bottom] in logs, screw treatment [control/unscrewed woods, screwed woods], and fungi species) with 5 replicates. Meanwhile, WL of screws, embedded into woods; and weight of rust powder (mg) that occurred due to screw-corrosion at screw-embedded woods were assessed using CRD with 2x3x8 factorial pattern (wood species, vertical part in the logs [top, middle, bottom], and fungi species), each similarly with 5 replicates. Further, average WL of woods in decay test and corroding test was grouped into 5 classes, which referred to class criteria for wood resistance against fungi attack, in accordance with SNI 7207:2014, i.e. class I-V. Further, data analysis on WL of screws (in fungi-medium) employed also CRD with single factor (eight fungi species) with 6 replicates. Finally, the fungi capability to decay woods was categorized into 5 stages, i.e. very low, low, moderate, high, and very high, which referred to criteria of wood loss following SNI 7207:2014's Standard. 


\section{RESULTS and DISCUSSION}

\subsection{Wood decay}

One of characteristics to measure decayed-woods by fungi is the weight-loss of wood, as it stated by Dinwoodie (1981) that wood weight-loss and wood damage indicate fungal attack on wood. Wood weightloss in decaying process reflects cell-wall destruction due to degradation process of wood chemical components particularly cellulose and lignin. Capability of particular fungi species in decaying particular wood species varies. Average weight loss of Parashorea spp. woods due to decaying-fungi is described in Table 1. The higher capability of particular fungi to decay woods, the lower the resistance of those woods.

Table 1 shows the variation capability of several fungi in decaying two Parashorea spp. Schizophyllum commune afforded the highest capability to decay woods which indicated by the greatest wood weight-loss, followed by Pycnoporus sanguineus. Meanwhile, lowest fungi capability to decay woods that indicated by the lowest wood weight-loss was performed by Lentinus lepideus followed by Chaetomium globosum. Further, Djarwanto (2018), Suprapti and Djarwanto (2012), and Takahashi and Kishima (1973) affirmed that in decaying woods, C. globosum and L. lepideus exhibited low capability, while Polyporus arcularius, Polyporus sp., Pycnoporus sanguineus, and Tyromyces palustris afforded high capability.

Tukey test $(\mathrm{p} \leq 0.05)$ revealed that overall average percentage weight-loss of Parashorea smythiesii (7.11\%) was lower than that of $P$. tomentella (9.86\%). One of the possible reasons is because of the presence of tyloses in the vessel cells of $P$. smythiesii (Djarwanto et al. 2014) which could inhibit the growth and spreading of fungi mycelium and consequently caused the slower process of wood degradation. De Micco et al. (2016) stated that the occlusion of vessel through tyloses or gums has important roles in limiting the spread of pathogens and wood decay organisms. Another previous study also mentioned the adverse effect of tyloses blocking in vessels on the balance between air and water which important for the growth of fungi (Quartey, 2009). Dickson (2000) indicated that the increase of elm trees resistance was influenced by the presence of tyloses.

Scrutinizing horizontal position of wood specimens in the logs, it is found that overall average weight-loss of heartwood corresponded to $6.20 \%$ (belonged to class III), which was lower compared to sapwood, i.e. $10.76 \%$ ( $\mathrm{p} \leq 0.05$ ) thereby regarded as a non-resistant wood group (class IV). This is due to the condition of tree samples, which were still in an active growth period and therefore physiologically developed more extractives thereby inhibiting fungi growth. Bamber (1987); Bouslimi et al. (2013); Takahashi and Kishima (1973) mentioned that extractive content in old wood was greater than in young wood, and also extractive content in heartwood was greater than in sapwood. Accordingly, the heartwood is more resistant against microorganism attack (including fungi) due to toxic-extractive presence. Further, Bouslimi et al. (2013); Suprapti and Djarwanto (2012) asserted that average weight loss of heartwood was greater than that of sapwood. This implies that resistance capability of heartwood is greater than sapwood. Meanwhile, scrutinizing vertical position in wood logs, it disclosed that the least weight loss occurred at the bottom part (7.42\%), followed in increasing order at the middle (8.57\%) and top part (9.46\%), as confirmed by Tukey test at $\mathrm{p} \leq 0.05$. It is understood that the proportion of mature wood at the bottom part was higher than the upper part of the stem.

The assessment of resistance class of woods against decaying-fungi through laboratory-scale showed that two Parashorea spp. could be classified into a moderatelyresistant wood group (class III). In these research results, Parashorea tomentella afforded greater resistance against organism attack than similar wood species, as 
Table 1. Average weight loss percentage and class resistance of woods against fungi

\begin{tabular}{|c|c|c|c|c|c|c|c|c|c|c|c|c|c|}
\hline \multirow{3}{*}{ Species } & \multicolumn{6}{|c|}{ Parashorea smythiesii (\%) } & \multicolumn{6}{|c|}{ Parashorea tomentella (\%) } & \multirow{3}{*}{$\begin{array}{c}\text { Overall } \\
\text { average } \\
\% \text { loss }(\%)\end{array}$} \\
\hline & \multicolumn{2}{|c|}{ Bottom } & \multicolumn{2}{|c|}{ Middle } & \multicolumn{2}{|c|}{ Top } & \multicolumn{2}{|c|}{ Bottom } & \multicolumn{2}{|c|}{ Middle } & \multicolumn{2}{|c|}{ Top } & \\
\hline & HW & SW & HW & SW & HW & SW & HW & SW & HW & SW & HW & SW & \\
\hline $\begin{array}{l}\text { Chaetomium } \\
\text { globosum }\end{array}$ & $\begin{array}{l}1.86^{\mathrm{BCDEFGH}} \\
\text { (II) }\end{array}$ & $\begin{array}{l}3.66^{\text {vwxyzABC }} \\
\text { DEFGH } \\
\text { (II) }\end{array}$ & $\begin{array}{l}1.98^{\text {ABCDEFG }} \\
{ }^{H} \text { (II) }\end{array}$ & $\begin{array}{l}4.42^{\text {uvwxyzAB }} \\
\text { CDEFGH (II) }\end{array}$ & $0.99^{\mathrm{FGH}}$ (II) & $\begin{array}{l}4.16^{\text {uwwxyzAB }} \\
\text { CDEFGH } \\
\text { (II) }\end{array}$ & $\begin{array}{l}1.69^{\mathrm{CDEFGH}} \\
\text { (II) }\end{array}$ & $\begin{array}{l}7.70^{\text {nopqrstuvw }} \\
\text { xyzABCDEF } \\
\text { (III) }\end{array}$ & $\begin{array}{l}2.74^{\text {xyzABCDE }} \\
\text { FGH (II) }\end{array}$ & $\begin{array}{l}8.18^{\text {nopqrstuvw }} \\
\text { xyzABCD }\end{array}$ & $\begin{array}{l}1.86^{\mathrm{BCDEFGH}} \\
\text { (II) }\end{array}$ & $\begin{array}{l}6.33^{\text {pqrstuvwxy }} \\
\text { zABCDEFGH } \\
\text { (III) }\end{array}$ & $\begin{array}{l}3.80^{\mathrm{f}}\{\mathrm{II} \\
(\mathrm{II}-\mathrm{III})\}\end{array}$ \\
\hline Lentinus lepideus & $0.63^{\mathrm{H}}$ (II) & $\begin{array}{l}4.83^{\text {turwxyzAB }} \\
\text { CDEFGH } \\
\end{array}$ & $\begin{array}{l}1.30^{\mathrm{EFGH}} \\
\text { (II) }\end{array}$ & $\begin{array}{l}3.75^{\text {vwxyzABC }} \\
\text { DEFGH } \\
\text { (II) }\end{array}$ & $0.96^{\mathrm{FGH}}$ (II) & $\begin{array}{l}4.99^{\text {tuvwxyzaB }} \\
\text { CDEFGH } \\
\text { (II) }\end{array}$ & $\begin{array}{l}1.32^{\mathrm{EFGH}} \\
\text { (II) }\end{array}$ & $\begin{array}{l}1.82^{\text {BCDEFGH }} \\
\text { (II) }\end{array}$ & $\begin{array}{l}1.36^{\text {DEFGH }} \\
\text { (II) }\end{array}$ & $\begin{array}{l}2.70^{\mathrm{yzABCDEF}} \\
\mathrm{GH} \text { (II) }\end{array}$ & $0.73^{\mathrm{GH}}$ (II) & $\begin{array}{l}2.83^{\text {xyzABCDE }} \\
\text { FGH } \text { (II) }\end{array}$ & $2.27^{\mathrm{g}}\{\mathrm{II}\}$ \\
\hline Phlebia brevispora & $\begin{array}{l}1.51^{\mathrm{CDEFGH}} \\
\text { (II) }\end{array}$ & $\begin{array}{l}4.89^{\text {turwxyzAB }} \\
\text { CDEFGH } \\
\end{array}$ & $\begin{array}{l}1.38^{\mathrm{DEFGH}} \\
\text { (II) }\end{array}$ & $\begin{array}{l}11.99^{\text {hijklmnop }} \\
\text { qrs } \text { (IV) }\end{array}$ & $\begin{array}{l}2.27^{A B C D E F G} \\
{ }^{H} \text { (II) }\end{array}$ & $\begin{array}{l}12.66^{\text {ghijklmno }} \\
{ }^{\mathrm{pq}}(\mathrm{IV})\end{array}$ & $\begin{array}{l}3.47^{\mathrm{vw} x y z A B C} \\
\text { DEFGH }\end{array}$ & $\begin{array}{l}5.67^{\text {rsturwxyzA }} \\
\text { BCDEFGH } \\
\end{array}$ & $\begin{array}{l}5.37^{\text {sturwxyza }} \\
\text { BCDEFGH } \\
\end{array}$ & $\begin{array}{l}7.03^{\text {qrstuvwxyz }} \\
\text { ABCDEFGH } \\
\text { (III) }\end{array}$ & $\begin{array}{l}4.61^{\text {uvwxyzAB }} \\
\text { CDEFGH }\end{array}$ & $\begin{array}{l}9.84^{\text {jklmnopqrst }} \\
\text { uvw } \text { (III) }\end{array}$ & $\begin{array}{l}5.89^{\mathrm{e}}\{\mathrm{III} \\
(\mathrm{II}-\mathrm{IV})\}\end{array}$ \\
\hline $\begin{array}{l}\text { Polyporus } \\
\text { arcularius }\end{array}$ & $0.66^{\mathrm{H}}$ (II) & $\begin{array}{l}15.09^{\text {efghijkl }} \\
\text { (IV) }\end{array}$ & $0.54^{\mathrm{H}}$ (II) & $\begin{array}{l}16.32^{\text {cdefghijk }} \\
\text { (IV) }\end{array}$ & $\begin{array}{l}3.53^{\text {vwxyzABC }} \\
\text { DEFGH } \\
\text { (II) }\end{array}$ & $\begin{array}{l}19.95^{\text {abcdefg }} \\
\text { (IV) }\end{array}$ & $\begin{array}{l}10.26^{\text {jklmnpqrs }} \\
\text { tuv }(\mathrm{IV})\end{array}$ & $\begin{array}{l}16.53^{\text {cdefghij }} \\
\text { (IV) }\end{array}$ & $\begin{array}{l}11.49^{\text {hijkklmnop }} \\
\text { qrst } \text { (IV) }\end{array}$ & $\begin{array}{l}12.97^{\mathrm{ijklmnop}} \\
\text { (IV) }\end{array}$ & $\begin{array}{l}8.05^{\text {nopqrstuvw }} \\
\text { xyzABCDE } \\
\text { (III) }\end{array}$ & $\begin{array}{l}14.45^{\text {efghijklm }} \\
{ }^{\mathrm{n}} \text { (IV) }\end{array}$ & $\begin{array}{l}10.82^{c}\{\text { IV } \\
\text { (II-IV) }\}\end{array}$ \\
\hline Polyporus sp. & $\begin{array}{l}8.12^{\text {nopqrstuvw }} \\
\text { xyzABCDE } \\
\text { (III) }\end{array}$ & $\begin{array}{l}7.92^{\text {opqrstuwwx }} \\
\text { yzABCDE }\end{array}$ & $\begin{array}{l}8.55^{\text {mnopqrstuv }} \\
\text { wxyzAB }\end{array}$ & $\begin{array}{l}6.14^{\text {qrstuvwxyz }} \\
\text { ABCDEFGH } \\
\text { (III) }\end{array}$ & $\begin{array}{l}2.63^{\mathrm{ZABCDEF}} \\
\mathrm{GH} \text { (II) }\end{array}$ & $\begin{array}{l}2.94^{\mathrm{xyzABCDE}} \\
\text { FGH } \\
\text { (II) }\end{array}$ & $\begin{array}{l}3.42^{\text {wxyzABCD }} \\
\text { EFGH (II) }\end{array}$ & $\begin{array}{l}9.36^{\text {Imnopqrstu }} \\
\text { vwxyz }\end{array}$ & $\begin{array}{l}4.68^{\text {tuwwxyzAB }} \\
\text { CDEFGH } \\
\end{array}$ & $\begin{array}{l}19.24^{\text {abcdefg }} \\
\text { (IV) }\end{array}$ & $\begin{array}{l}5.94^{\text {qrsturwxyz }} \\
\text { ABCDEFGH } \\
\text { (III) }\end{array}$ & $\begin{array}{l}15.66^{\text {efghijkl }} \\
\text { (IV) }\end{array}$ & $\begin{array}{l}7.89^{d}\{\mathrm{III} \\
(\mathrm{II}-\mathrm{IV})\}\end{array}$ \\
\hline $\begin{array}{l}\text { Pycnoporus } \\
\text { sanguineus }\end{array}$ & $\begin{array}{l}2.53^{\mathrm{zABCDEF}} \\
\mathrm{GH} \text { (II) }\end{array}$ & $\begin{array}{l}7.51^{\text {opqrstuwwx }} \\
\text { yzABCDEFG } \\
\text { (III) }\end{array}$ & $\begin{array}{l}3.36^{\text {wxyzABCD }} \\
\text { EFGH } \\
\text { (II) }\end{array}$ & $\begin{array}{l}9.36^{\text {Imnopqrstu }} \\
\text { vwxyz } \\
\text { (III) }\end{array}$ & $\begin{array}{l}12.84^{\text {ghijklmno }} \\
{ }^{\mathrm{p}} \text { (IV) }\end{array}$ & $24.29^{\mathrm{ab}}(\mathrm{IV})$ & $\begin{array}{l}6.43^{\text {pqrasturwxy }} \\
\text { zABCDEFGH } \\
\text { (III) }\end{array}$ & $\begin{array}{l}15.87^{\text {defghijkl }} \\
\text { (IV) }\end{array}$ & $\begin{array}{l}15.68^{\text {efghijkl }} \\
\text { (IV) }\end{array}$ & $\begin{array}{l}22.79^{\mathrm{abc}} \\
\text { (IV) }\end{array}$ & $\begin{array}{l}8.74^{\text {mnopqrstuv }} \\
\text { wyzA } \\
\text { (III) }\end{array}$ & $\begin{array}{l}17.66^{\text {bcdefghi }} \\
\text { (IV) }\end{array}$ & $\begin{array}{l}12.25^{\mathrm{b}}\{\mathrm{IV} \\
(\mathrm{II}-\mathrm{IV})\}\end{array}$ \\
\hline $\begin{array}{l}\text { Schizophyllum } \\
\text { commune }\end{array}$ & $\begin{array}{l}12,06^{\text {hijkkmnop }} \\
\text { qrs (IV) }\end{array}$ & $\begin{array}{l}12.41^{\text {hijklmnop }} \\
\text { qr }(\text { IV) }\end{array}$ & $\begin{array}{l}10.91^{\text {ijklmnopq }} \\
\text { rstu }(\mathrm{IV})\end{array}$ & $\begin{array}{l}8.31^{\text {nopqrstuvw }} \\
\text { xyzABC } \\
\text { (III) }\end{array}$ & $\begin{array}{l}11.49^{\mathrm{ijklmnopq}} \\
{ }_{\mathrm{rst}}(\mathrm{IV})\end{array}$ & $\begin{array}{l}21.15^{\text {abcde }} \\
\text { (IV) }\end{array}$ & $22.6^{\text {abcd }}$ (IV) & $\begin{array}{l}17.69^{\text {bcdefghi }} \\
\text { (IV) }\end{array}$ & $25.43^{\mathrm{a}}(\mathrm{IV})$ & $\begin{array}{l}17.79^{\text {bcdefgh }} \\
\text { (IV) }\end{array}$ & $\begin{array}{l}14.05^{\text {fghijklmn }} \\
\text { (IV) }\end{array}$ & $\begin{array}{l}17.78^{\text {bcdefgh }} \\
\text { (IV) }\end{array}$ & $\begin{array}{l}15.97^{\mathrm{a}}\{\mathrm{IV} \\
(\mathrm{III-IV})\}\end{array}$ \\
\hline Tyromyces palustris & $\begin{array}{l}4.19^{\text {uvwxyzaB }} \\
\text { CDEFGH }\end{array}$ & $\begin{array}{l}2.57^{\mathrm{ZABCDEF}} \\
\mathrm{GH} \text { (II) }\end{array}$ & $\begin{array}{l}4.57^{\text {uvwxyzaB }} \\
\text { CDEFGH } \\
\text { (II) }\end{array}$ & $\begin{array}{l}3.43^{\text {wxyzABCD }} \\
\text { EFGH } \\
\text { (II) }\end{array}$ & $\begin{array}{l}20.06^{\text {abcdef }} \\
\text { (IV) }\end{array}$ & $\begin{array}{l}9.51^{\text {klmnopqrst }} \\
\text { uvwy } \\
\text { (III) }\end{array}$ & $\begin{array}{l}5.96^{\text {qrsturwxyz }} \\
\text { ABCDEFGH } \\
\text { (III) }\end{array}$ & $\begin{array}{l}17.10^{\text {cdefghi }} \\
\text { (IV) }\end{array}$ & $\begin{array}{l}9.55^{\text {klmnopqrst }} \\
\text { uvwx } \\
\text { (III) }\end{array}$ & $\begin{array}{l}10.99^{\mathrm{ijk} / m n o p q} \\
\text { rstu } \\
\text { (IV) }\end{array}$ & $\begin{array}{l}3.56^{\text {vwxyzABC }} \\
\text { DEFGH } \\
\text { (II) }\end{array}$ & $\begin{array}{l}16.40^{\text {cdefghij }} \\
\text { (IV) }\end{array}$ & $\begin{array}{l}8.99^{d}\{\text { III } \\
(\text { II-IV) }\}\end{array}$ \\
\hline $\begin{array}{l}\text { Average \% } \\
\text { loss of HW (\%) }\end{array}$ & 4.95 \{IV (II & II-IV) $\}$ & & & & & $7.46\{$ III & (II-IV)\} & & & & & 6.20 \\
\hline $\begin{array}{l}\text { Average } \% \\
\text { loss of SW (\%) }\end{array}$ & 9.26 \{III (II & I-IV)\} & & & & & $12.26\{$ IV & $V($ II-IV) $\}$ & & & & & 0.76 \\
\hline $\begin{array}{l}\text { Overall average } \\
\% \text { loss (\%) }\end{array}$ & $7.11^{\mathrm{b}}\{\mathrm{III}$ & (II-IV)\} & & & & & $9.86^{a}\{\mathrm{III}$ & II (III-IV)\} & & & & & \\
\hline
\end{tabular}

mentioned in the previous report by Oey (1990), which conversely belonged to class IV, whereby he made an evaluation based on life service of woods, without mentioning specific species of attacking-organisms. According to Martawijaya and Barly (2010), woods that belonged to class I-II could be used for construction materials. However, if the woods belonged to class III-IV, they should be treated or preserved prior to their uses to increase their resistance against attackingorganisms. Djarwanto (2018) stated that those woods could be preserved with CCB (copper-chrome-boron) and CCF (copper-chrome-fluor) chemicals using soaking or vacuum-pressure methods.

\subsection{The decay of screwed woods}

Average weight loss of wood that embedded by screws; weight loss of screws, inserted into woods; and weight of rust powder (collected from the former screw holes at screw-embedded woods) are presented in Tables 2, 3, 4. Analysis using CRD revealed that wood species, screw treatment, and fungi species affected wood weight-loss, screw weight-loss, and weight of rust powder significantly $(\mathrm{p} \leq 0.05)$. The greatest weight loss of woods (19.73\%) occurred in Parashorea tomentella taken from the middle part of the logs, which were embedded with screws and then exposed to Schizophyllum commune (Table 2). Fungi attack on woods which were 
Table 2. Average percentage weight loss and their resistance class of heartwood after embedded by screw and exposed to fungi

\begin{tabular}{|c|c|c|c|c|c|c|c|c|c|c|c|c|c|}
\hline \multirow{3}{*}{ Species } & \multicolumn{6}{|c|}{ Parashorea smythiesii (\%) } & \multicolumn{6}{|c|}{ Parashorea tomentella (\%) } & \multirow{3}{*}{$\begin{array}{c}\text { Overall } \\
\text { average \% } \\
\text { loss (\%) }\end{array}$} \\
\hline & \multicolumn{2}{|c|}{ Bottom } & \multicolumn{2}{|c|}{ Middle } & \multicolumn{2}{|c|}{ Top } & \multicolumn{2}{|c|}{ Bottom } & \multicolumn{2}{|c|}{ Middle } & \multicolumn{2}{|c|}{ Top } & \\
\hline & Screw & No & Screw & No & Screw & No & Screw & No & Screw & No & Screw & No & \\
\hline $\begin{array}{l}\text { Chaetomium } \\
\text { globosum }\end{array}$ & $\begin{array}{l}3.48^{\text {opq }} \\
\text { rsturwxyz } \\
\text { (II) }\end{array}$ & $\begin{array}{l}1.80^{\text {tuvwxyz }} \\
\text { (II) }\end{array}$ & $\begin{array}{l}6.49^{\mathrm{ijklmnop}} \\
\text { qrst } \\
\text { (III) }\end{array}$ & $\begin{array}{l}1.89^{\text {stuvwxyz }} \\
\text { (II) }\end{array}$ & $\begin{array}{l}2.90^{\text {opqrstuv }} \\
\text { wxyz (II) }\end{array}$ & $\begin{array}{l}1.04^{\text {wxyz }} \\
\text { (II) }\end{array}$ & $\begin{array}{l}3.39^{\text {opqrstuv }} \\
\text { wxyz } \\
\text { (II) }\end{array}$ & $\begin{array}{l}1.43^{\text {uvwxyz }} \\
\text { (II) }\end{array}$ & $\begin{array}{l}4.14^{\text {mnopqrst }} \\
\text { uvwxyz }\end{array}$ & $\begin{array}{l}2.58^{\text {pqrstuvw }} \\
\text { xyz } \text { (II) }\end{array}$ & $\begin{array}{l}3.73^{\text {nopqrstuv }} \\
\text { wxyz } \\
\text { (II) }\end{array}$ & $\begin{array}{l}1.64^{\mathrm{tuvwxyz}} \\
\text { (II) }\end{array}$ & $\begin{array}{l}2.88^{d}\{\mathrm{II} \\
(\mathrm{II}-\mathrm{III})\}\end{array}$ \\
\hline $\begin{array}{l}\text { Lentinus } \\
\text { lepideus }\end{array}$ & $\begin{array}{l}1.05^{\mathrm{wxyz}} \\
\text { (II) }\end{array}$ & $0.59^{\mathrm{z}}$ (II) & $\begin{array}{l}1.06^{\text {wxyz }} \\
\text { (II) }\end{array}$ & $\begin{array}{l}1.17^{\mathrm{vwxyz}} \\
\text { (II) }\end{array}$ & $0.34^{\mathrm{z}}$ (II) & $0.93^{x y z}$ (II) & $\begin{array}{l}1.41^{\mathrm{uvw} x y z} \\
\text { (II) }\end{array}$ & $\begin{array}{l}1.27^{\mathrm{uvwxyz}} \\
\text { (II) }\end{array}$ & $\begin{array}{l}7.61^{\text {ghijklmn }} \\
\text { opq }(\text { III) }\end{array}$ & $\begin{array}{l}1.18^{\mathrm{vwxyz}} \\
\text { (II) }\end{array}$ & $\begin{array}{l}1.28^{\mathrm{uvwxyz}} \\
\text { (II) }\end{array}$ & $0.68^{\mathrm{z}}$ (II) & $\begin{array}{l}1.55^{\mathrm{e}}\{\mathrm{II} \\
(\mathrm{II}-\mathrm{III})\}\end{array}$ \\
\hline $\begin{array}{l}\text { Phlebia } \\
\text { brevispora }\end{array}$ & $0.55^{\mathrm{z}}$ (II) & $\begin{array}{l}1.49^{\mathrm{uvw} w y z} \\
\text { (II) }\end{array}$ & $\begin{array}{l}1.16^{\mathrm{vwxyz}} \\
\text { (II) }\end{array}$ & $\begin{array}{l}1.35^{\mathrm{uvwxyz}} \\
\text { (II) }\end{array}$ & $\begin{array}{l}2.71^{\text {pqrstuvw }} \\
\text { xyz } \text { (II) }\end{array}$ & $\begin{array}{l}2.23^{\text {stuvwxyz }} \\
\text { (II) }\end{array}$ & $\begin{array}{l}3.88^{\text {nopqrstuv }} \\
\text { wxyz } \\
\text { (II) }\end{array}$ & $\begin{array}{l}3.24^{\text {opqrstuv }} \\
\text { wxyz (II) }\end{array}$ & $\begin{array}{l}6.92^{\text {hijklmno }} \\
\text { pqrs } \\
\text { (III) }\end{array}$ & $\begin{array}{l}5.10^{\mathrm{lmn} n o p q r s} \\
\text { turwxyz }\end{array}$ & $\begin{array}{l}5.07^{\mathrm{lmn} n o p q r s} \\
\text { turwxyz }\end{array}$ & $\begin{array}{l}4.34^{\text {mnopqrst }} \\
\text { uvwxyz } \text { (II) }\end{array}$ & $\begin{array}{l}3.17^{\mathrm{d}}\{\mathrm{II} \\
(\mathrm{II}-\mathrm{IV})\}\end{array}$ \\
\hline $\begin{array}{l}\text { Polyporus } \\
\text { arcularius }\end{array}$ & $\begin{array}{l}1.66^{\mathrm{tuvwxyz}} \\
\text { (II) }\end{array}$ & $0.74^{\mathrm{yz}}$ (II) & $\begin{array}{l}1.90^{\text {stuvwxyz }} \\
\text { (II) }\end{array}$ & $0.51^{\mathrm{z}}$ (II) & $\begin{array}{l}7.44^{\text {ghijklmn }} \\
\text { opqr } \text { (III) }\end{array}$ & $\begin{array}{l}3.52^{\text {nopqrstuv }} \\
\text { wxyz } \\
\text { (II) }\end{array}$ & $\begin{array}{l}3,56^{\text {nopqrstuv }} \\
\text { wxyz (II) }\end{array}$ & $\begin{array}{l}10.27^{\text {efghijkl }} \\
\text { (IV) }\end{array}$ & $\begin{array}{l}6.15^{\mathrm{klmnnopqr}} \\
\text { stuvwx }\end{array}$ & $\begin{array}{l}11.24^{\text {efghijk }} \\
\text { (IV) }\end{array}$ & $\begin{array}{l}6.42^{\mathrm{ijk} k \mathrm{mnop}} \\
\text { qrstuv } \\
\text { (III) }\end{array}$ & $\begin{array}{l}7.74^{\text {ghijklmn }} \\
\text { op (III) }\end{array}$ & $\begin{array}{l}5.10^{c}\{\text { III } \\
(\text { II-IV) }\}\end{array}$ \\
\hline Polyporus sp. & $\begin{array}{l}12.48^{\text {defg }} \\
\text { (IV) }\end{array}$ & $\begin{array}{l}6.25^{j \mathrm{klmnopq}} \\
\text { rstuvw } \\
\text { (III) }\end{array}$ & $\begin{array}{l}12.47^{\text {defg }} \\
\text { (IV) }\end{array}$ & $\begin{array}{l}6.09^{\text {klmnopqr }} \\
\text { stuvwx } \\
\text { (III) }\end{array}$ & $\begin{array}{l}8.01^{\text {ghijklmno }} \\
\text { (III) }\end{array}$ & $\begin{array}{l}3.28^{\text {opqrstuv }} \\
\text { wxyz } \\
\text { (II) }\end{array}$ & $\begin{array}{l}4.32^{\text {mnopqrst }} \\
\text { uvwxyz } \text { (II) }\end{array}$ & $\begin{array}{l}3.90^{\text {nopqrstuv }} \\
\text { wxyz } \\
\text { (II) }\end{array}$ & $\begin{array}{l}17.36^{\mathrm{abcd}} \\
\text { (IV) }\end{array}$ & $\begin{array}{l}4.95^{\text {nopqrstuv }} \\
\text { wxyz (II) }\end{array}$ & $\begin{array}{l}13.56^{\text {cdef }} \\
\text { (IV) }\end{array}$ & $\begin{array}{l}6.13^{\text {klmnopqr }} \\
\text { stuvwx } \\
\text { (III) }\end{array}$ & $\begin{array}{l}8.23^{\mathrm{b}}\{\mathrm{III} \\
(\mathrm{II}-\mathrm{IV})\}\end{array}$ \\
\hline $\begin{array}{l}\text { Pycnoporus } \\
\text { sanguineus }\end{array}$ & $\begin{array}{l}2.82^{\text {opqrstuv }} \\
\text { wxyz } \\
\text { (II) }\end{array}$ & $\begin{array}{l}2.45^{\text {qrstuvwx }} \\
\text { yz (II) }\end{array}$ & $\begin{array}{l}2.24^{\text {stuvwxyz }} \\
\text { (II) }\end{array}$ & $\begin{array}{l}3.33^{\text {opqrstuv }} \\
\text { wxyz (II) }\end{array}$ & $\begin{array}{l}2.06^{\text {stuvwxyz }} \\
\text { (II) }\end{array}$ & $\begin{array}{l}10.80^{\text {efghijk }} \\
\text { (IV) }\end{array}$ & $\begin{array}{l}3.59^{\text {nopqrstuv }} \\
\text { wxyz } \\
\text { (II) }\end{array}$ & $\begin{array}{l}6.52^{\mathrm{ijk} k \mathrm{mnop}} \\
\text { qrstu } \\
\text { (III) }\end{array}$ & $\begin{array}{l}6.48^{\mathrm{ijk} k \mathrm{mnop}} \\
\text { qrstu } \\
\text { (III) }\end{array}$ & $\begin{array}{l}14.42^{\text {bcde }} \\
\text { (IV) }\end{array}$ & $\begin{array}{l}1.10^{\text {wxyz }} \\
\text { (II) }\end{array}$ & $\begin{array}{l}8.77^{\text {fghijklmn }} \\
\text { (III) }\end{array}$ & $\begin{array}{l}5.38^{c}\{\mathrm{III} \\
(\mathrm{II}-\mathrm{IV})\}\end{array}$ \\
\hline $\begin{array}{l}\text { Schizophyllum } \\
\text { commune }\end{array}$ & $\begin{array}{l}6.89^{\text {hijklmno }} \\
\text { pqrs } \\
\text { (III) }\end{array}$ & $\begin{array}{l}9.33^{\text {efghijklm }} \\
\text { (III) }\end{array}$ & $\begin{array}{l}11.99^{\mathrm{efgh}} \\
(\mathrm{IV})\end{array}$ & $\begin{array}{l}9.42^{\text {efghijklm }} \\
\text { (III) }\end{array}$ & $\begin{array}{l}12.42^{\text {defg }} \\
\text { (IV) }\end{array}$ & $\begin{array}{l}11.47^{\text {efghi }} \\
\text { (IV) }\end{array}$ & $\begin{array}{l}11.54^{\text {efghi }} \\
\text { (IV) }\end{array}$ & $\begin{array}{l}17.36^{\mathrm{abcd}} \\
\text { (IV) }\end{array}$ & $\begin{array}{l}19.73^{\mathrm{a}} \\
\text { (IV) }\end{array}$ & $\begin{array}{l}19.17^{\mathrm{ab}} \\
(\mathrm{IV})\end{array}$ & $\begin{array}{l}18.37^{\mathrm{abc}} \\
\text { (IV) }\end{array}$ & $\begin{array}{l}14.03^{\text {bcdef }} \\
\text { (IV) }\end{array}$ & $\begin{array}{l}13.48^{a} \\
\{\text { IV } \\
(\text { III-IV) }\}\end{array}$ \\
\hline $\begin{array}{l}\text { Tyromyces } \\
\text { palustris }\end{array}$ & $\begin{array}{l}11.67^{\mathrm{efghi}} \\
\text { (IV) }\end{array}$ & $\begin{array}{l}4.23^{\text {mnopqrst }} \\
\text { uvwxyz } \text { (II) }\end{array}$ & $\begin{array}{l}14.11^{\text {bcde }} \\
\text { (IV) }\end{array}$ & $\begin{array}{l}4.28^{\text {mnopqrst }} \\
\text { uvwxyz } \text { (II) }\end{array}$ & $\begin{array}{l}7.12^{\text {hijklmno }} \\
\text { pqrs } \\
\text { (III) }\end{array}$ & $\begin{array}{l}6.41^{\mathrm{ijklmnop}} \\
\text { qrstuv } \\
\text { (III) }\end{array}$ & $\begin{array}{l}10.57^{\text {efghijk }} \\
\text { (IV) }\end{array}$ & $\begin{array}{l}5.98^{\text {klmnopqr }} \\
\text { stuvwxy } \\
\text { (III) }\end{array}$ & $\begin{array}{l}13.41^{\text {cdef }} \\
\text { (IV) }\end{array}$ & $\begin{array}{l}9.34^{\text {efghijklm }} \\
\text { (III) }\end{array}$ & $\begin{array}{l}18.05^{\mathrm{abc}} \\
\text { (IV) }\end{array}$ & $\begin{array}{l}3.60^{\text {nopqrstuv }} \\
\text { wxyz } \\
\text { (II) }\end{array}$ & $\begin{array}{l}9.06^{\mathrm{b}}\{\mathrm{III} \\
(\mathrm{II}-\mathrm{IV})\}\end{array}$ \\
\hline $\begin{array}{l}\text { Average \% loss } \\
\text { of screwed (\%) }\end{array}$ & 5.63 & & & & & & 7.99 & & & & & & 6.81 \\
\hline $\begin{array}{l}\text { Averae \% loss } \\
\text { of unscrewed (\%) }\end{array}$ & 3.94 & & & & & & 6.87 & & & & & & 5.41 \\
\hline $\begin{array}{l}\text { Overall average } \\
\% \text { loss }(\%)\end{array}$ & $4.78^{\mathrm{b}}\{\mathrm{II}$ & (II-IV)\} & & & & & $7.42^{a}\{\mathrm{III}$ & I (II-IV)\} & & & & & \\
\hline
\end{tabular}

screwed could also cause corrosion to screws. The presence of fungi and its metabolism process imparted a significant role on screw corrosion. Metals would prevalently become corroded, when in physical contact with humid air, acid, base, salt solutions, and oils that contained water (Hendrix, 2006). Further, the resistance and safety of building frames are often determined by the fastening between metals and woods (Li et al., 2011). Corrosion of metals which are fastened to woods could also trigger wood decay (Xu, 2017).

Average weight loss of screwed and unscrewed (control) wood at Parashorea tomentella (7.99\% and 6.87\%) is greater than that of P. smythiesii (5.63\% and $3.94 \%$, respectively). Further, the overall average weight loss of unscrewed woods (5.41\%) was signi- ficantly lower than screwed woods (6.81\%). This was commensurate with the statement by Suprapti and Djarwanto (2015), who asserted that weight loss of woods fastened by screws was greater compared to woods without screws. This situation revealed that screws fastened to woods could also affect the wooddecay rate. Further, Noetzli et al. (2007) also affirmed that the presence of screws in physical contact with woods could enhance fungi activity to decay woods. Based on the Tukey test $(\mathrm{p} \leq 0.05)$ revealed that the overall average percentage weight-loss of Parashorea smythiesii (4.78\%) was lower than that of P. tomentella (7.42\%), as on Table 2. It reveals greatest overall average weight loss of woods ( $\mathrm{p} \leq 0.05$ ), which were embedded by screws occurred at the middle part of logs $(7.16 \%)$, 
followed by the top part (6.16\%), while lowest weight loss was at the bottom part of logs (4.99\%).

Each fungi species afforded varying capability in decaying screw-embedded heartwood; in corroding screws embedded into woods. Schizophyllum commune exhibited the highest in decaying two species of Parashorea spp woods, embedded by screws. Meanwhile, four fungi species (Polyporus arcularius, Polyporus sp., Pycnoporus sanguineus, and Tyromyces palustris) afforded moderate capability. Ultimately, Chaetomium globosum, Lentinus lepideus, and Phlebia brevispora fungi revealed low capability.

The $\mathrm{pH}$ of two Parashorea spp. wood species varied about 6.01-6.40, conforming the statements by Takahashi and Kishima (1973), \& Li et al. (2011), woods with $\mathrm{pH}$ in 4.5-7.0 range tended to enhance metal-corrosion potential. Further, Cole and Schofield (2000) asserted that prevalently woods with $\mathrm{pH}$ equal to 4 or less were corrosive to metals than with $\mathrm{pH}$ above 5 .

\subsection{Screw corrosion in wood and fungal culture media}

Table 3 reveals that greatest weight loss of screws that fastened into woods occurred at the bottom of $P$. tomentella wood-logs, further exposed to Schizophyllum commune fungi. Relevantly, Krilov (1987) asserted that metals which were fastened to different wood species exhibited differing metal weight-losses as well. According to Knaebe (2013), woods with high extractive content tended to inflict corrosion more severely on irons. Weight loss of screws occurred because reaction proceeded between wood extractives and metals, which caused metals corrosion and hence weight loss. Besides, such reaction also caused woods in physical contact with screws to undergo hydrolysis and hence decrease wood weight. According to Foliente, Leicester, Ang, Mackenzie, and Cole (2018), initially, corrosion damaged metals, fastened to woods; gradually destroyed; and then negatively-affected wood resistance. Further, Kip and van-Veen (2015) explained that corrosion of screws fastened to woods implied the impact of serial reaction that resulted from chemicals, physicals, and microbiology reaction processes, further inducing wood decay. Besides, biocorrosion indicates the consequences of electrochemical reaction affected by microorganisms (including fungi).

From the Table 3, it can be seen that the overall average weight loss of screw $(\mathrm{p} \leq 0.05)$ at $P$. tomentella (1.75\%) is greater than that of $P$. smythiesii (1.24\%). Further, overall average weight loss of screws each exhibited insignificant difference $(\mathrm{p} \leq 0.05)$ among different vertical positions in wood logs i.e. bottom (1.54\%), middle (1.36\%), and top portions (1.61\%).

Greatest fungi capability in corroding the screws which embedded into woods was found at Polyporus arcularius (2.78\%). Besides that, the greatest capability in corroding screws (placed on fungi-culture media) was performed by Schizophyllum commune (8.54\%), while the capability of seven fungi to corrode metals were not significantly different from each other $(\mathrm{p} \leq$ 0.05). In relevant, Suhartanti (2006) stated that fungi afforded great role in corroding metals. Further, it is revealed that weight loss of screws (embedded into woods) was mostly lower than the loss of screws (in fungi culture-media). This is because in the latter case, fungi activity could solely exclusively corrode the screws, but not in the former case.

\subsection{Rust powder in wood}

Table 4 discloses weight data of rust powder, leftbehind inside screw holes after screws' removal from decayed-woods. The greatest weight of rust powder was encountered inside former screw holes of screw-removed decayed-wood specimens originated from the bottom of Parashorea tomentella wood logs, then placed on Schizophyllum commune fungi culture; followed by decreasing order by top part, placed on the same culture. Weight of rust powder as formed due to Schizophyllum 
Determining the Wood (Parashorea spp.) Decaying and Metal Corroding Abilities of Eight Fungi

Table 3. Weight loss percentage of the screws embedded into woods and in fungal culture media

\begin{tabular}{|c|c|c|c|c|c|c|c|c|}
\hline \multirow{2}{*}{ Species } & \multicolumn{3}{|c|}{ Parashorea smythiesii } & \multicolumn{3}{|c|}{ Parashorea tomentella } & \multicolumn{2}{|c|}{$\begin{array}{l}\text { Overall average } \\
\% \text { loss (\%) }\end{array}$} \\
\hline & Bottom & Middle & Top & Bottom & Middle & Top & In wood & $\begin{array}{l}\text { In culture } \\
\text { media }\end{array}$ \\
\hline Chaetomium globosum & $0.75^{\text {fghij }}$ & $0.91^{\text {fghij }}$ & $0.44^{\text {ghij }}$ & $0.21^{\mathrm{ij}}$ & $0.99^{\text {fghij }}$ & $0.50^{\text {ghij }}$ & $0.63^{e}$ & $4.36^{\mathrm{b}}$ \\
\hline Lentinus lepideus & $0.10^{\mathrm{j}}$ & $0.28^{\text {hij }}$ & $0.71^{\text {fghij }}$ & $1.08^{\text {fghij }}$ & $0.77^{\text {fghij }}$ & $1.83^{\text {bcdefghij }}$ & $0.79^{e}$ & $3.48^{\mathrm{b}}$ \\
\hline Phlebia brevispora & $0.99^{\text {fghij }}$ & $0.80^{\text {fghij }}$ & $1.77^{\text {cdefghij }}$ & $1.78^{\text {cdefghij }}$ & $3.27^{\mathrm{abc}}$ & $1.83^{\text {bcdefghij }}$ & $1.74^{\mathrm{bc}}$ & $3.34^{\mathrm{b}}$ \\
\hline Polyporus arcularius & $3.11^{\mathrm{abcd}}$ & $1.46^{\text {defghij }}$ & $2.00^{\text {bcdefgh }}$ & $2.97^{\text {abcde }}$ & $3.55^{\mathrm{ab}}$ & $3.56^{\mathrm{ab}}$ & $2.78^{\mathrm{a}}$ & $2.86^{\mathrm{b}}$ \\
\hline Polyporus sp. & $1.35^{\text {efghij }}$ & $0.30^{\mathrm{hij}}$ & $2.04^{\text {bcdefgh }}$ & $1.03^{\text {fghij }}$ & $1.12^{\text {fghij }}$ & $0.47^{\text {ghij }}$ & $1.05^{\mathrm{de}}$ & $2.81^{\mathrm{b}}$ \\
\hline Pycnoporus sanguineus & $1.05^{\text {fghij }}$ & $1.48^{\text {defghij }}$ & $0,87^{\text {fghij }}$ & $1.88^{\text {bcdefghi }}$ & $2.19^{\text {abcdefg }}$ & $0.84^{\text {fghij }}$ & $1.38^{\mathrm{cd}}$ & $2.51^{\mathrm{b}}$ \\
\hline Schizophyllum commune & $1.52^{\text {cdefghij }}$ & $0.58^{\text {ghij }}$ & $2.44^{\text {abcdef }}$ & $3.95^{\mathrm{a}}$ & $1.31^{\text {efghij }}$ & $3.56^{\mathrm{ab}}$ & $2.23^{\mathrm{b}}$ & $8.54^{\mathrm{a}}$ \\
\hline Tyromyces palustris & $1.52^{\text {cdefghij }}$ & $1.99^{\text {bcdefgh }}$ & $1.54^{\text {cdefghij }}$ & $1.41^{\text {defghij }}$ & $0.73^{\text {fghij }}$ & $1.35^{\text {efghij }}$ & $1.41^{\mathrm{cd}}$ & $0.94^{\mathrm{b}}$ \\
\hline $\begin{array}{l}\text { Average \% loss of } \\
\text { bottom part }\end{array}$ & & 1.30 & & & 1.79 & & & 1.54 \\
\hline $\begin{array}{l}\text { Average \% loss of } \\
\text { middle part }\end{array}$ & & 0.98 & & & 1.74 & & & 1.36 \\
\hline $\begin{array}{l}\text { Average \% loss of } \\
\text { top part }\end{array}$ & & 1.48 & & & 1.74 & & & 1.61 \\
\hline $\begin{array}{l}\text { Overall average \% } \\
\text { loss (\%) }\end{array}$ & & $1.24^{\mathrm{b}}$ & & & $1.75^{\mathrm{a}}$ & & & \\
\hline
\end{tabular}

Table 4. Weight of rust powder (mg) in post-screwed holes

\begin{tabular}{|c|c|c|c|c|c|c|c|}
\hline \multirow{2}{*}{ Species } & \multicolumn{3}{|c|}{ Parashorea smythiesii (mg) } & \multicolumn{3}{|c|}{ Parashorea tomentella (mg) } & \multirow{2}{*}{$\begin{array}{c}\text { Overall } \\
\text { average } \\
\text { weight }(\mathrm{mg})\end{array}$} \\
\hline & Bottom & Middle & Top & Bottom & Middle & Top & \\
\hline Chaetomium globosum & $3.6^{\text {op }}$ & $3.4^{\text {op }}$ & $3.0^{\mathrm{p}}$ & $3.2^{p}$ & $2.8^{p}$ & $2.6^{\mathrm{p}}$ & $3.1^{\mathrm{e}}$ \\
\hline Lentinus lepideus & $27.8^{\text {abcdefghi }}$ & $15.8^{\text {fghijklmnop }}$ & $25.6^{\text {bcdefghijk }}$ & $19.6^{\text {efghijklmn }}$ & $26.2^{\text {abcdefghij }}$ & $30.0^{\text {abcdefg }}$ & $24.17^{\mathrm{b}}$ \\
\hline Phlebia brevispora & $12.4^{\mathrm{ijklmnop}}$ & $11.8^{\mathrm{jklmnop}}$ & $10.8^{\mathrm{jklmnop}}$ & $23.4^{\text {cdefghijkl }}$ & $24.4^{\text {bcdefghijk }}$ & $23.0^{\text {cdefghijklm }}$ & $17.63^{\mathrm{c}}$ \\
\hline Polyporus arcularius & $25.8^{\text {abcdefghij }}$ & $38.4^{\mathrm{abc}}$ & $28.8^{\text {abcdefg }}$ & $22.6^{\text {defghijklm }}$ & $25.0^{\text {bcdefghijk }}$ & $23.6^{\text {cdefghijkl }}$ & $27.37^{\mathrm{ab}}$ \\
\hline Polyporus sp. & $8.4^{\operatorname{lmnop}}$ & $3.0^{p}$ & $1.2^{\mathrm{p}}$ & $20.6^{\text {efghijklmn }}$ & $19.0^{\text {efghijklmno }}$ & $7.4^{\text {mnop }}$ & $9.93^{\mathrm{d}}$ \\
\hline Pycnoporus sanguineus & $5.4^{\text {nop }}$ & $12.8^{\text {hijklmnop }}$ & $16.8^{\text {fghijklmnop }}$ & $15.6^{\text {ghijklmnop }}$ & $36.6^{\mathrm{abcd}}$ & $5.0^{\text {nop }}$ & $15.37^{\mathrm{c}}$ \\
\hline Schizophyllum commune & $22.0^{\text {defghijklm }}$ & $22.0^{\text {defghijklm }}$ & $31.4^{\text {abcdef }}$ & $41.4^{\mathrm{a}}$ & $33.6^{\text {abcde }}$ & $39.6^{\mathrm{ab}}$ & $31.67^{\mathrm{a}}$ \\
\hline Tyromyces palustris & $5.6^{\text {nop }}$ & $2.0^{\mathrm{p}}$ & $10.0^{\mathrm{klmnop}}$ & $29.6^{\text {abcdefg }}$ & $28.4^{\text {abcdefgh }}$ & $19.4^{\text {efghijklmn }}$ & $15.83^{\mathrm{c}}$ \\
\hline $\begin{array}{l}\text { Average \% loss of } \\
\text { bottom part }\end{array}$ & & 13.88 & & & 22.00 & & 17.94 \\
\hline $\begin{array}{l}\text { Average \% loss of } \\
\text { middle part }\end{array}$ & & 13.65 & & & 24.50 & & 19.08 \\
\hline $\begin{array}{l}\text { Average \% loss of } \\
\text { top part }\end{array}$ & & 15.95 & & & 18.83 & & 17.39 \\
\hline $\begin{array}{l}\text { Overall average } \\
\text { weight (mg) }\end{array}$ & & $14.49^{\mathrm{b}}$ & & & $21.78^{\mathrm{a}}$ & & \\
\hline
\end{tabular}

Remarks: Numbers in the column as well as in the row followed horizontally, and overall average weight loss values in each column and row followed by same letters are not significantly different (Tukey test, $\mathrm{p} \leq 0.05$ ). 
commune fungi was the greatest (31.67 mg), followed by decreasing order by Polyporus arcularius (Table 4). Based on the Tukey test $(\mathrm{p} \leq 0.05)$, the overall average weight rust powder of Parashorea smythiesii (14.49 mg) was lower than that of $P$. tomentella $(21.78 \mathrm{mg})$. The overall average weight of rust powder exhibited insignificant variation $(\mathrm{p} \leq 0.05)$ among different vertical positions in wood logs i.e. bottom (17.94 mg), middle (19.08 mg), and top portions (17.39 mg).

Greatest fungi capability in corroding the screws placed on fungi-culture media was performed by Schizophyllum commune. In relevant, Suhartanti (2006) stated that fungi afforded great role in corroding metals. Weight of rust powder as formed due to Schizophyllum commune fungi was the greatest, followed in decreasing order by Polyporus arcularius (Table 4). Further, it is shown that weight loss of screws (embedded into woods) was mostly lower than the loss of screws (in fungi culture-media). This is because in the latter case, fungi activity could solely exclusively (100\%) corrode the screws, but not in the former case.

\section{CONCLUSION}

The capability of fungi to decay $P$. smythiesii and $P$. tomentella beginning from the highest to the lowest was consecutively Schizophyllum commune, Pycnoporus sanguineus, Polyporus arcularius, Tyromyces palustris, Polyporus sp., Phlebia brevispora, Chaetomium globosum, and ultimately Lentinus lepideus fungi. For each Parashorea species, the average weight loss of heartwood (belonged to class III) was lower than sapwood (class IV). Meanwhile, vertically, the average weight loss at the bottom part of the wood was the lowest.

On the other hand, average weight loss of woods which were fastened by screws was higher than woods without screws. Fungi capability in decaying screwembedded woods from the highest until lowest was consecutively S. commune, T. palustris, Polyporus sp.,
P. sanguineus, $P$. arcularius, $P$. brevispora, C. globosum and ultimately $L$. lepideus. Greatest corrosion of screws, embedded into woods was caused by $P$. arcularius activity. Further, the greatest weight of rust powder at post-screw holes decayed-woods was caused by $S$. commune activity, while the effect of other seven fungi species was almost similar to each other.

\section{ACKNOWLEDGMENT}

The authors would like to thank Forest Products Research and Development Center, Research Development and Innovation Agency, Indonesian Ministry of Environment and Forestry for funding this research.

\section{REFERENCES}

An, J.E., Lee, S.Y., Ryu, S.H., Kim, M. 2018. Transformation of terpene synthase from Polyporus brumalis in Pichia pastoris for recombinant enzyme production. Journal of the Korean Wood Science and Technology 46(4): 415-422.

Bouslimi, B., Koubaa, A., Bergeron, Y. 2013. Variation of brown rot decay in eastern white cedar (Thuja occidenalis L.). BioResources 8(3): 4735-4755.

Bamber, R.K. 1987. Sapwood and heartwood. Technical Publication number 2. Revised. Forestry Commission of New South Wales.

Cole, H.G., Schofield, M.J. 2000. Corrosion of metals by wood. In Corrosin Eds L.L. Shreir, R.A. Jarman \& G.T. Burstein. Butterworth-Heinemann, Linacre House, Yordan Hill, Oxford OX2 8DP, Great Britain.

De Micco, V., Balzano, A., Wheeler, E.A., Baas, P. 2016. Tyloses and gums: a review of structure, function and occurrence of vessel occlusions. IAWA Journal 37(2): 186-205. doi: 10.1163/22941932-20160130. 
Dickson, W. C. 2000. Integrative Plant Anatomy. Academic press. A Harcourt Science and Technology company. 525 B Street, Suite 1900, San Diego, California 92101-4495, USA. pp. 533.

Dinwoodie, J.M. 1981. Timber, Its Nature and Behaviour. Van Nostrand Reinhold Co. Ltd, USA.

Djarwanto, Suprapti, S. 2015. Corrosion properties on five wood species from Sukabumi in the open site to metal screw. Journal of Tropical Wood Science and Technology 13(2): 136-145.

Djarwanto. 2018. Wood Decay Fungi and Forest Resource Conservation. Research Professor Inauguration Book. Research, Development and Innovation Agency, Jakarta.

Djawanto, Suprapti, S., Hutapea, F.J. 2018. The capability of ten fungus strains in decaying four wood species from Manokwari. Journal of Forest Products Research 36(2): 129-138.

Djawanto, Dewi, L.M., Muslich, M., Jasni, Suprapti, S., Pari, G., Hadjib, N., Indrawan, D.A., Basri, E. 2014. Basic properties and utilization of wood species from Kalimantan. Annual research report. Forest Products Research and Development Center, Bogor, Indonesia.

Foliente, G.C., Leicester, R.H., Ang, C.H., Mackenzie, C., Cole, I. 2018. Durability design of wood construction. Retrieved from https:/www.reseachgate. net/publication/ 283234837, accessed on 7 January 2019.

Hendrix, P.E. 2006. Corrosion on metals in contact with preservative treated wood an update. Retrieved from www.archchemicals.com/Fed/wolw/Docs/ corrosion-of-metals-606, accessed on 28 April 2013.

Kip, N., Van-Veen, J.A.. 2015. Mini review, the dual role of microbes in corrosion. The ISME Journal 9: 542-551.

Knaebe, M. 2013. Iron stain on wood. Finisline Forest Products Laboratory. USDA Forest Service, Madison.
Retrieved from www.fpl.fs.fed.us, accessed on 9 November 2019.

Krilov, A. 1987. Corrosive properties of some Eucalypts. Wood Science and Technology 21: 211-217.

Lee, S.Y., Ryu, S.H., Choi, I.G., Kim, M. 2016. Biosynthesis of eudesmane-type sesquiterpenoids by the wood-rotting fungus, Polyporus brumalis, on specific medium, including inorganic magnesium source. Journal of the Korean Wood Science and Technology 44(2): 253-263.

Li, Z.W., Marston, N.J., Jones, M.S. 2011. Corrosion of fasteners in treated timber. Branz study report 241. Branz Ltd, Judgeford, New Zealand.

Martawijaya, A., Barly. 2010. Pedoman pengawetan kayu untuk mengatasi jamur dan rayap pada bangunan rumah dan gedung. Bogor: IPB Press.

Noetzli, K.P., Frey, A.B.B., Graf, F., Holdenrieder, T.S.O. 2007. Release of iron from bonding nails in torrent control check dams and its effect on wood decomposition by Fomitopsis pinicola. Wood Res. 52: 47-60.

Oey, D. S. 1990. Specific Gravity of Indonesia Woods and Its Significance for Practical Use. Communication Nr.3. Forest Products Research and Development Center, Bogor, Indonesia.

Pawlik, A., Ruminowicz-Stefaniuk, M., Frąc, M., Mazur, A., Wielbo, J., Janusz, G. 2019. The wood decay fungus Cerrena unicolor adjusts its metabolism to grow on various types of wood and light conditions. PLoS ONE. Public Library of Science 14(2): 1-19. p.e0211744.

Soerianegara, I., Lemmens, R. 1993. Plant Resources of Southeast Asia 5(1), Timber Trees: Major Commercial Timbers. PROSEA, Indonesia.

Sugianto, K., Sudika, D.A. 2010. Metal corrosion in waterborne preservative-treated wood. Journal of Forestry Research 7: 91-99.

Suhartanti D. 2006. Corrosion rate of steel by Desulfomicrobium baculatum and Desulfomonas 
pigra. Berkala MIPA 16: 27-35.

Suprapti, S., Djarwanto. 2012. The resistance of six wood species against decaying fungi. Journal of Forest Products Research 30(3): 227-234. ISSN 0216-4329.

Suprapti, S., Djarwanto. 2014. The resistance of five wood species from Ciamis against eleven strain of decaying fungi. Journal of Forest Products Research 32(3): 189-198.

Suprapti, S., Djarwanto, Andianto. 2016. Decay resistance of six wood species from Papua against destroying fungi. Journal of Forest Products Research 34(2): 157-165.

SNI (Indonesian National Standard). 2014. Wood Resis- tance Test Against Wood Destroying Organism, No. SNI 7207:2014. Badan Standarisasi Nasional, Jakarta.

Takahashi, M., Kishima, T. 1973. Decay resistance of sixty-five Southeast Asian timber specimens in accelerated. Tonan Ajia Kenkyu 10(4): 525-541.

Quartey, G.A. 2009. Relationships between some anatomical, physical and durability properties of the wood of some lesser utilised Ghanaian hardwoods. Ph.D Thesis, Kwame Nkrumah University of Science and Technology, Kumasi, Ghana.

Xu X. 2017. Corrosion of embedded ferrous metals in woods. Retrieved from www.corrosionguru.com. p.: 1-9, accessed on 20 July 2018. 